\title{
Low level laser therapy for sports injuries
}

\author{
Yusuke Morimoto ${ }^{1}$,Akiyoshi Saito ${ }^{1}$, Yasuaki Tokuhashi ${ }^{2}$ \\ 1: Surugadai Nibon University Hospital, Department of Orthopaedic Surgery, Tokyo, Japan \\ 2: Nibon University School of medicine, Department of Orthopaedic Surgery, Tokyo, Japan
}

\begin{abstract}
Background and aims: Our hospital has used LLLT in the treatment of athletes since 1990. We had a good result about LLLT for sports injuries. However, few articles have attempted to evaluate the efficacy of LLLT for sports injuries. The aims of this study was to evaluate the efficacy of LLLT for sports injuries.

Materials (Subjects) and Methods: Forty one patients underwent LLLT in our hospital. These patients included 22 men and 19 women with an average age of 38.9 years old. Patients were irradiated by diode laser at points of pain and/or acupuncture points. Patients underwent LLLT a maximum treatment of 10 times (mean 4.1 times). We evaluated the efficacy of LLLT using a Pain relief score (PRS). A score of 2 to 5 after treatment was regarded as very good, 6 to 8 as good, and 9 to 10 as poor. A PRS score of less than 5 was regarded as effective.

Results: The rate of effectiveness (PRS of 5 or less) after LLLT was 65.9\% (27/41 patients).

Discussion: In this study, the resulting rate of effectiveness was $65.9 \%$ for all sports injuries. However, we have a high rate of effectiveness for Jumper's knee, tennis elbow and Achilles tendinitis and cases that were irradiated laser by a physician.

Conclusions: LLLT is an effective treatment for sports injuries, particularly jumper's knee, tennis elbow and Achilles tendinitis.
\end{abstract}

Key words: Low level laser therapy (LLLT) - Sports injuries

\section{Introduction}

It is not easy to control pain caused by overuse injuries what athletes have during sports activities. Because, athletes are not able to take enough rest for treatment caused by continued training.

Low Level Laser Therapy (LLLT) has been used for sports injuries and conditioning for many years 1,2 ). Our hospital has used LLLT in the treatment of athletes since 1990. When LLLT was first started at our hospital, a diode laser with an output of 150 milliwatts ( $\mathrm{mW}$ ). Next, the machine of a diode laser with an output of 1 watt (W). At the beginning, we used a diode laser with an output of between $150 \mathrm{~mW}$ and $1 \mathrm{~W}$. To our knowledge, efficacious sports injuries indicating LLLT are bursitis and inflammation of the tendon sheath, for example lateral and medial epicondylitis of the

Addressee for Correspondence:

Yusuke Morimoto MD PhD

Department of Orthopaedic Surgery, Surugadai Nihon

University Hospital

1-8-13, Kanda Surugadai, Chiyoda, Tokyo, Japan

Telephone: +81-3-3293-1711 FAX: +81-3-3292-0307

E-mail: morimoto.yuusuke@nihon-u.ac.jp humerus, patellar tendinitis, pes anserinus bursitis, plantar fasciitis, Achilles peritenonitis, etc. The usual output level in recent years has risen to $10 \mathrm{~W}$ from 2006. We feel that $10 \mathrm{~W}$ LLLT is effective for treatment of sports injuries better than $150 \mathrm{~mW}$ and $1 \mathrm{~W}$ LLLT.

The advantages of LLLT for athletes are: noninvasive procedure, fewer complications, no complications for athletes conscious of drug testing, and simple administration. As described above, we believe $10 \mathrm{~W}$ LLLT is a useful tool for sports injuries. However, few articles have attempted to evaluate the efficacy of $10 \mathrm{~W}$ LLLT for sports injuries.

The purpose of this study was to evaluate the efficacy of LLLT for sports injuries.

\section{Materials and methods}

\section{Patients}

One hundred and twenty four patients underwent LLLT

Received Date: January 5th, 2013

Accepted Date: January 26th, 2013

J-STAGE Advance Publication Date: February 10th, 2013 
since December 2006 at our institute. Sixty seven of the 124 patients were treated with LLLT for sports injuries. Of these, 41 patients underwent LLLT 2 or more times. These patients included 22 men and 19 women with an average age of $38.9 \pm 15.9$ (from 17 to 77 ) years old. These 41 patients were included in our study.

Injuries included jumper's knee, lateral and medial epicondylitis of the humerus, Achilles tendinitis, injury of adductor muscle, rotator cuff injury, etc. (table 1) The sports in which patients participated included golf, judo, basketball, marathon, aerobics, skiing, softball, dance, tennis, triathlon, volleyball, etc. (table 2) Some patients took medicines which were non-steroidal anti-inflammatory drugs or a poultice a few times.

Table 1: Sports injuries contained in this study

\begin{tabular}{|l|c|}
\hline \multicolumn{1}{|c|}{ injuries } & cases \\
\hline Jumper' s knee & 8 \\
Lateral epicondylitis of humerus & 3 \\
Achilles tendinitis & 3 \\
Adductor muscle injury & 2 \\
Medial epicondylitis of humerus & 2 \\
Rotator cuff injury & 2 \\
etc & 21 \\
\hline
\end{tabular}

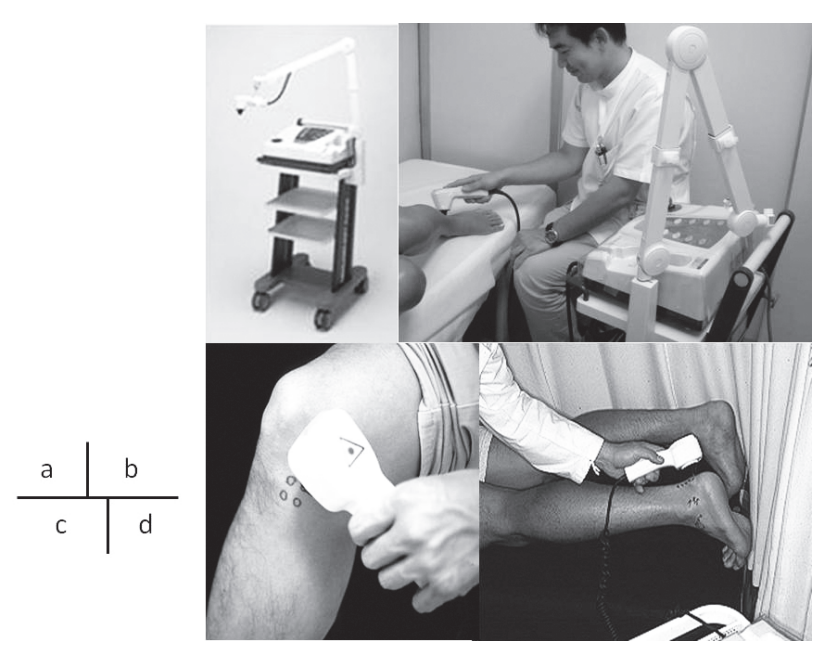

Figure 1 a: diode laser (Medilaser soft pulse 10), b: physician irradiation, $\mathbf{c}, \mathbf{d}$ : irradiation at pain point

\section{Laser irradiation}

A medilaser soft pulse 10 (Gallium-Alminium-Arsenide Diode LASER, MATSUSHITA INDUSTRIAL EQUIPMENT CO., LTD) was used for this study. (figure 1-a) The wave length of this machine was $830 \mathrm{~nm}$, output power was $10 \mathrm{~W}$, and power density was $6-7 \mathrm{~W} / \mathrm{cm}^{2}$. Patients were irradiated by laser at points of pain (figure 1-c.d) and/or acupuncture points. (figure 2) We used acupuncture point irradiation for chronic pain or neuralgia, selected some acupuncture points respectively. Laser irradiation was administered for 10 or 15 seconds at each point in 5 to 10 minutes in a single weekly treatment. Patients underwent LLLT 2 or more times with a maximum treatment of 10 times (mean 4.1 times) either by physician (figure 1-b) or by self-treat-

Table 2: Sports in which patients participated

\begin{tabular}{|l|c|}
\hline \multicolumn{1}{|c|}{ sports } & cases \\
\hline golf & 6 \\
martial arts & 4 \\
basketball & 4 \\
marathon & 3 \\
aerobics & 2 \\
ski & 2 \\
softball & 2 \\
dance & 2 \\
tennis & 2 \\
triathlon & 2 \\
volleyball & 2 \\
etc & 10 \\
\hline
\end{tabular}

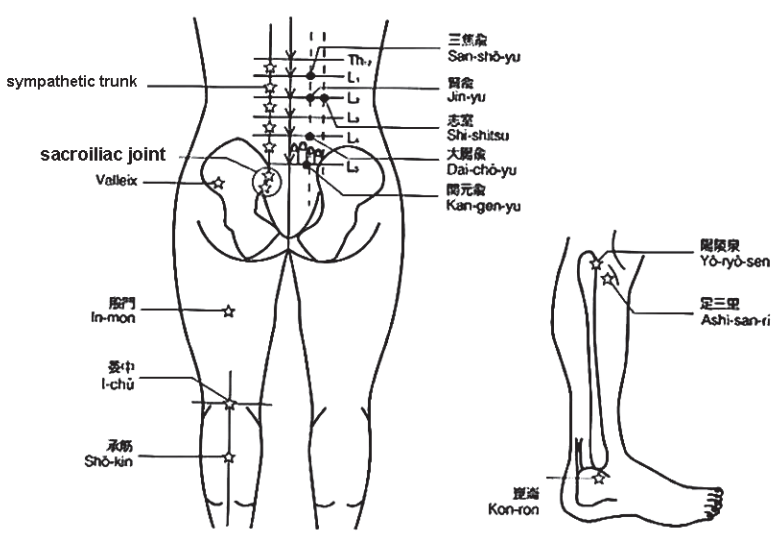

Figure 2: Acupuncture points 
ment.

We evaluated the efficacy of LLLT using a Pain relief score (PRS) which was proposed by Japan Laser Therapy Association ( JALTA ) ${ }^{3)}$. A pain score before treatment is defined 10 points. After treatment, a patient evaluates PRS by comparison with Pain score before treatment. If a patient has no pain after treatment, PRS is 0. If a patient has half pain after treatment, PRS is 5. A PRS score of 0 to 1 after treatment was regarded as excellent. A score of 2 to 5 after treatment was regarded as very good, 6 to 8 as good, and 9 to 10 as poor. A PRS score of less than 5 was regarded as effective. (table 3)

\section{Results}

The resulting Pain relief scores after LLLT were as follows (figure 3): A score of 0 was recorded in 9 patients, 1 in 2 patients, 2 in 3 patients, 3 in 7 patients, 4 in 4 patients, 5 in 2 patients, 6 in 6 patients, 7 in 2 patients, 8 in 3 patients, 9 in no patients, and 10 in 3

Table 3: Pain Relief Score (PRS)

\begin{tabular}{|c|c|c|}
\hline $\begin{array}{c}\text { improvement } \\
\text { of pain score }\end{array}$ & evaluation & determination \\
\hline $10 \rightarrow 0 \sim 1$ & excellent & \multirow{2}{*}{ effective } \\
\hline $10 \rightarrow 2 \sim 5$ & very good & \\
\hline $10 \rightarrow 6 \sim 8$ & good & \multirow{2}{*}{ not effective } \\
\hline $10 \rightarrow 9 \sim 10$ & poor & \\
\hline $10 \rightarrow 11 \sim$ & worse & \\
\hline
\end{tabular}

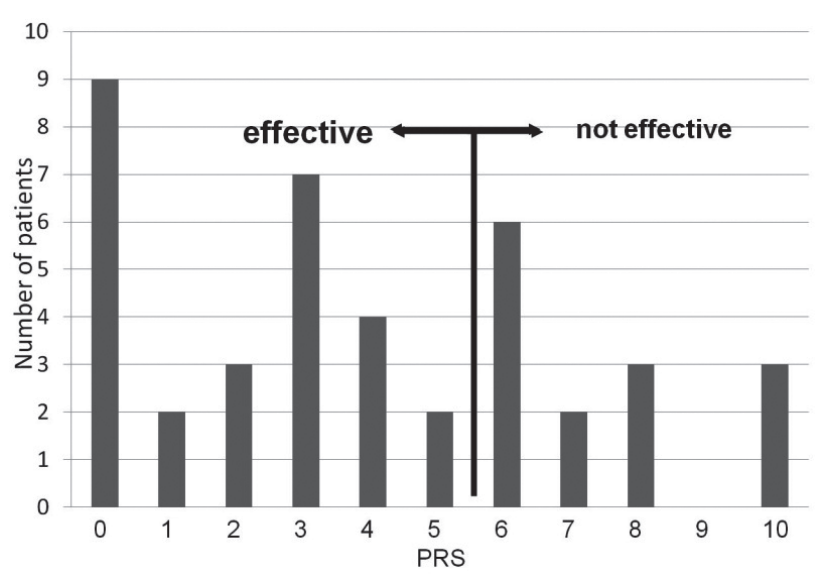

Figure 3: PRS after LLLT patients. The rate of effectiveness (a score of 5 or less) was $65.9 \%$ (27/41 patients). The following injuries resulted in higher rates of effectiveness: $75 \%$ for jumper's knee, $66.6 \%$ for tennis elbow, and $66.6 \%$ for Achilles tendinitis.

\section{Discussion}

\section{Mechanism of LLLT 4,5)}

LLLT is known to have many bioavailable effects. LLLT activates the electron transport chain, increases ATP synthesis, and therefore causes macrophage, fibroblast and lymphocyte activity. Some studies have shown that LLLT changes nerve conduction and regeneration and induces vasodilation, particularly in microcirculation. The effect of pain relief through LLLT is caused by the development of circulation in the injured area. Pain producing substances are washed out by vascular flow, regardless of the type of pain.

LLLT is clinically useful for pain relief. The side effects and complications of LLLT are unreported. For athletes conscious of drug testing, it is of great benefit. For athletes, such as professional athletes, who are unable to take proper rest to heal injuries, LLLT can be administered daily and in short treatment sessions.

\section{Methods of irradiation of laser}

Many irradiation methods have been documented. Irradiation on tender points has an effect on shallow disorders, and irradiation on acupuncture points has an effect on deep disorders (lumbar disc herniations, etc.) or on complicating pain (rotator cuff injury, etc.).

In this study, LLLT was administered on both tender points and acupuncture points. The resulting rate of effectiveness, $65.9 \%$, was not particularly good. However, the rate of effectiveness in cases of irradiation by a physician was 100\%. Psychological influences and improper irradiation technique of patients engaging in self-treatment may partially account for the low rate of effectiveness.

Jumper's knee, lateral epicondylitis of humerus and Achilles tendinitis are injuries commonly treated for long periods of time. It is difficult to manage these disorders. The results of our study show a higher rate of effectiveness in patients with these disorders, and we believe that LLLT can be a useful treatment.

While LLLT has been shown to be effective, longterm treatment is often impractical. Patients are required to come to the hospital for treatment at frequent intervals. High cost prevents teams or individual athletes from buying the necessary equipment. In addi- 
tion, there is a high risk of improper irradiation technique for athletes who choose administer LLLT themselves.

The limitations of this study were the small number of patients and the subjective grading method by the patients. More objective studies should be performed in the future.

\section{References}

1: Ohshiro T, Okada T, Ito E, Maeda T (1986): Diode Laser Treatment for Sports Pain by Comparison of Thermography, the Second Report. Journal of Japan Society for Laser Surgery and Medicine, 6(3): 383-386.

2: Ishide $\mathrm{Y}$, Ohshiro T, Ueda F, Murayama M, Ohshiro T, Fujii S, Takenouchi K, Kohzuma M (2008) : The Effect of GaAlAs Diode Laser on PreSports Warmingup and Post-sports Cooling Down. Laser Therapy, 17(4): 187-192.

3: Oshiro T (2000): The Evolution of the Japan Laser

\section{Conclusions}

Forty-one patients with sports injuries were treated by using LLLT.

The rate of effectiveness was $65.9 \%$. The rate of effectiveness in cases of jumper's knee, tennis elbow and Achilles tendinitis was high. LLLT is an effective treatment for sports injuries, particularly jumper's knee, tennis elbow and Achilles tendinitis.

Therapy Association (JALTA) Methods and Standard for Scoring Laser Therapy Pain Attenuation: A Retrospective Two-year Overview. Laser Therapy, 13 (1): 65-72.

4: http://www.photobiology.info/Hamblin.html

5: Saito K, Katagiri T, Ogawa M, Matsumoto S, Kubota J, Ohshiro T, Tsukamoto T, Okumura T, Nakaji S, Umeda T (2005): Effects of Diode Laser Irradiation on Superficial Blood Flow in College Sumo Wrestlers: A Preliminary Study. Laser Therapy, 14(2): 83-86. 